\title{
New perspectives on gravity wave remote sensing by spaceborne infrared limb imaging
}

\author{
P. Preusse ${ }^{1}$, S. Schroeder ${ }^{1}$, L. Hoffmann ${ }^{1}$, M. Ern ${ }^{1}$, F. Friedl-Vallon ${ }^{2}$, J. Ungermann ${ }^{1}$, H. Oelhaf ${ }^{2}$, H. Fischer ${ }^{2}$, and \\ M. Riese ${ }^{1}$ \\ ${ }^{1}$ Institute of Chemistry and Dynamics of the Geosphere (ICG-1), Forschungszentrum Jülich, Jülich, Germany \\ ${ }^{2}$ Institute for Meteorology and Climate Research, Forschungszentrum Karlsruhe, Karlsruhe, Germany
}

Received: 16 January 2009 - Published in Atmos. Meas. Tech. Discuss.: 17 March 2009

Revised: 10 June 2009 - Accepted: 18 June 2009 - Published: 25 June 2009

\begin{abstract}
Gravity wave (GW) remote sensing from space now has reached a stage of maturity that some first confinements for GW modeling can be deduced. This is in particular due to global distributions of absolute values of GW momentum flux from infrared limb sounders and due to 2-D maps of the horizontal wave field provided by nadir viewing instruments. The logical step forward is an infrared limb imager (ILI) which combines the good vertical resolution of limb sounding with horizontal mapping capabilities and provides 3-D images of the GW temperature structures. In this paper we investigate 1) how an ILI advances measurements of GW momentum flux, 2) which additional benefits are achieved by limb imaging of GWs, and 3) how an ILI compares to other GW momentum flux measurements, in-situ, ground-based, and from space. In particular, the large advance made by gaining regular 3-D sampling is demonstrated.
\end{abstract}

\section{Introduction}

Gravity waves (GWs) are an important coupling process conveying momentum from the troposphere into the middle and upper atmosphere. It has long been recognized that primarily GWs cause the wind reversal in the upper mesosphere, the cold summer mesopause and the mesospheric vortex break down (McLandress, 1998). They contribute to the stratospheric Brewer-Dobson circulation and provide the majority of the wave drag driving the summer time branch (Alexander and Rosenlof, 2003) and they contribute about half of

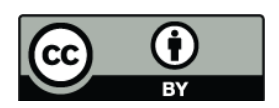

Correspondence to: P. Preusse (p.preusse@fz-juelich.de) the momentum driving the quasi-biennial oscillation (QBO) (Dunkerton, 1997). The QBO, in turn, strongly influences mixing processes between tropics and extratropics in the stratosphere (Shuckburgh et al., 2001) and the reaction of the atmosphere on the solar cycle (Mayr et al., 2006, 2007). Recent modeling results indicate that GWs are important for tropospheric variability in climate change (Sigmond et al., 2007,2008 ) and contribute at least one third to the acceleration of the Brewer-Dobson circulation, which is a predicted consequence of anthropogenic climate change (McLandress and Shepherd, 2008).

Gravity waves are mesoscale waves. Their horizontal wavelengths range from a few $10 \mathrm{~km}$ to the order of $1000 \mathrm{~km}$. This makes them challenging to measure from space. However, only satellite instruments can provide the global coverage above both land and oceans, which is required to estimate the global effects of GWs on the atmosphere mentioned above. Preusse et al. (2008) discuss which horizontal and vertical wavelengths of GWs are important for driving the middle atmosphere. They also show the sub-ranges of this wavelength regime covered by the different spaceborne measurement techniques and review our current current knowledge of the relative importance of these sub-ranges. Here we focus on the quality of the deduced GW parameters and how limb imaging can provide the fundamental advance from absolute values to direction resolved gravity wave momentum flux.

Very recently, space based measurements reached a maturity that they actually can be used to constrain GW parameterizations or global models capable to resolve part of the GW spectrum. For instance, Ern et al. (2006) used CRISTA measurements of the absolute value of GW momentum flux to constrain tuneable parameters of the Warner and McIntyre

Published by Copernicus Publications on behalf of the European Geosciences Union. 


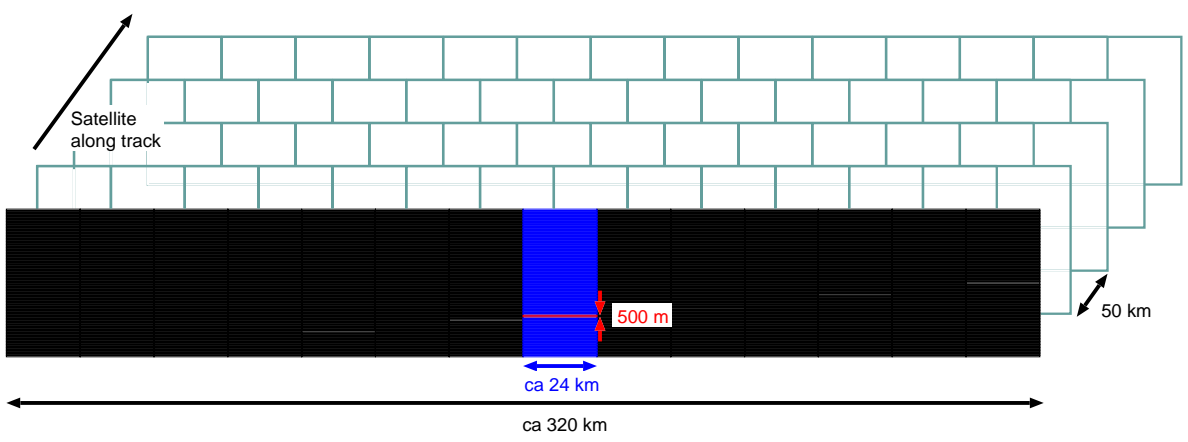

Fig. 1. Three-dimensional sampling of the discussed infrared limb imager. In the dynamics mode interferograms are taken every $50 \mathrm{~km}$ along the orbital track. The vertical extent of a single element is about $500 \mathrm{~m}$. Measurements are taken in $14 \mathrm{columns}$ of each $24 \mathrm{~km}$ width, resulting in 14 parallel tracks of altitude profiles.

GW parameterization scheme (Warner and McIntyre, 2001). Eckermann et al. (2006) validated a gravity wave resolving general circulation model (GCM), a mesoscale model and a dedicated mountain wave model by microwave nadir measurements of a mountain wave above the Scandinavian mountain ridge. Both examples demonstrate that resolving horizontal wave structures is essential. The next logical step is therefore to resolve the wave fronts in all three dimensions, i.e. map the horizontal wave structures (as done by nadir sounding instruments) at the good vertical resolution provided by the limb sounding instruments. On a case study basis this can be approached by combining the data from nadir and limb viewing satellite instruments (Alexander et al., 2008a). However, the two types of instruments observe very different parts of the GW spectrum with only a small overlap (Preusse et al., 2008) and for a systematic survey we therefore need to measure horizontal and vertical wave structure by the same instrument. This can be obtained by an infrared limb imager (ILI). Recent advances in detector technology now make ILIs technically feasible (Friedl-Vallon et al., 2006).

The relevant technical parameters for the ILI considered in this study are illustrated in Fig. 1. The vertical coverage is $48 \mathrm{~km}$ and the horizontal coverage $320 \mathrm{~km}$. The spatial sampling in vertical direction is $0.5 \mathrm{~km}$, in horizontal direction it is $24 \mathrm{~km}$. These data are taken simultaneously by the imaging technique resulting in 14 parallel tracks of vertical profiles. The along track sampling distance is $50 \mathrm{~km}$. The sampling geometry has been chosen in acccordance with our experiences from deducing GW momentum flux from CRISTA and the conclusions drawn by Ern et al. (2004). The spectral sampling of the instrument is $1.25 \mathrm{~cm}^{-1}$ in two spectral bands from 770 to $980 \mathrm{~cm}^{-1}$ and from 1070 to $1650 \mathrm{~cm}^{-1}$. The sensitivity is assumed to be better than $1 \mathrm{nW} /\left(\mathrm{cm}^{2} \mathrm{srcm}^{-1}\right)$ in the relevant spectral regions.

A general overview of the scientific objectives benefitting particularly from this new technology is given by Riese et al. (2005), and in a companion paper by Adams et al. (2008) we discuss the use of oversampling clouds by an inherent, high resolution cloud measuring mode sampling every $500 \mathrm{~m}$ in vertical direction and every $4 \mathrm{~km}$ across-track.

In the current paper we describe how an ILI can revolutionize space based GW measurements. The paper is organized as follows. In Sect. 2 we describe how GW momentum flux can be calculated from temperature measurements. We describe the leading error sources of current generation measurements and demonstrate how a limb imager can largely reduce or completely avoid such uncertainties. In Sect. 3 we discuss, how the regularly sampled, three-dimensional data of an ILI will advance measurement-modeling comparison and GW-source identification by GW backtracing. Section 4 compares different techniques to measure GW momentum flux, including in-situ and ground based measurements. It is discussed which techniques can be used to validate the results obtained by an ILI. It is also shown that an ILI is our only option to gain global distributions of GW momentum flux for a wide spectral range of GWs and for a wider altitude range. Finally, we will summarize the results and give an outlook, which new analysis methods will have to be developed to fully exploit this new technology.

\section{Momentum flux inference and limitations of current satellite measurements}

The vertical flux of the horizontal pseudomomentum $\left(F_{P x}, F_{P y}\right)$ carried by a $\mathrm{GW}$ is given by Fritts and Alexander (2003)

$$
\left(F_{P x}, F_{P y}\right)=\bar{\rho}\left(1-\frac{f^{2}}{\hat{\omega}^{2}}\right)\left(\overline{u^{\prime} w^{\prime}}, \overline{v^{\prime} w^{\prime}}\right),
$$

where $\bar{\rho}$ is the density of the background atmosphere, $f$ is the Coriolis parameter, $\hat{\omega}$ is the intrinsic GW frequency and $\left(u^{\prime}, v^{\prime}, w^{\prime}\right)$ is the wind perturbation in $(x, y, z)$ direction caused by the GW. The optimal method to measure GW momentum flux would therefore be to measure spectra of all three wind velocity components $(u, v, w)$ in a reference system moving with the background wind $(\bar{u}, \bar{v})$. We will 
discuss in Sect. 4 how close any given instruments, groundbased, in-situ or spaceborne, can fulfill this aim. One of the chief difficulties is that typical vertical wind velocities $w^{\prime}$ in the stratosphere are smaller than $1 \mathrm{~ms}^{-1}$. This makes direct measurements of $\left(\overline{u^{\prime} w^{\prime}}, \overline{v^{\prime} w^{\prime}}\right)$ from space infeasible. However, to circumvent this difficulty, GW momentum flux can also be inferred from temperature measurements.

Based on GW polarization relations Ern et al. (2004) have deduced an equivalent expression for $\mathrm{GW}$ induced temperature amplitudes. They also note that for typical cases in satellite measurements the deviation from the mid-frequency solution $(f \ll \hat{\omega} \ll N)$ is less than $10 \%$, often much smaller. In the mid frequency case $\mathrm{GW}$ pseudomomentum flux equals GW momentum flux. For briefness we therefore refer below only to GW momentum flux and give here the mid-frequency solution:

$$
\left(F_{P x}, F_{P y}\right)=\frac{1}{2} \bar{\rho}\left(\frac{g}{N}\right)^{2}\left(\frac{\hat{T}}{T}\right)^{2} \times\left(\frac{k}{m}, \frac{l}{m}\right)
$$

where $(k, l)=\left(2 \pi / \lambda_{x}, 2 \pi / \lambda_{y}\right)$ is the horizontal wave vector of the GW, $m=2 \pi / \lambda_{z}$ is the vertical wavenumber and $\lambda$ the wavelength in the respective direction. Further, $T$ is the background temperature, $\hat{T}$ the temperature amplitude of the $\mathrm{GW}, N$ the buoyancy frequency, and $g$ the gravity acceleration. Thus, if one is able to determine the full wave vector and the temperature amplitude of a GW or the respective wave vectors and temperature amplitudes of a superposition of GWs one can infer GW momentum flux.

It sometimes has been claimed that GW polarization relations may not hold. If this were true in general, it would pose an extremely serious problem, because almost all measurements (cf. Sect. 4) and all GW parameterization schemes rely on the polarization relations and linear wave theory (e.g. for calculating the onset of GW breaking). However, to us no stringent evidence for such claims is known. Instead, often more questionable assumptions on, e.g., the shape of the wave spectrum have to be made, since the measurements are insufficient to provide a full characterization of the GW. An ILI combined with other methods (measurement and modeling) could provide well-founded conditions for testing the validity of linear wave theory as discussed in Sect. 3 .

\subsection{Main error sources for current estimates}

The measurement geometry of infrared limb sounding and how it affects GW remote sensing is discussed in detail by Preusse et al. (2002). Here we only give a brief overview. The geometry for one limb ray is shown in Fig. 2 using a coordinate system with origin at Earth's center. Note that we use an aspect ratio of 1 between $\mathrm{x}$ and $\mathrm{y}$ axis. The instrument views slightly downward towards the Earths horizon, throughout the atmosphere and into space again. The origin of the radiation along the ray path is given in the upper panel. For optically thin conditions, this weighting function

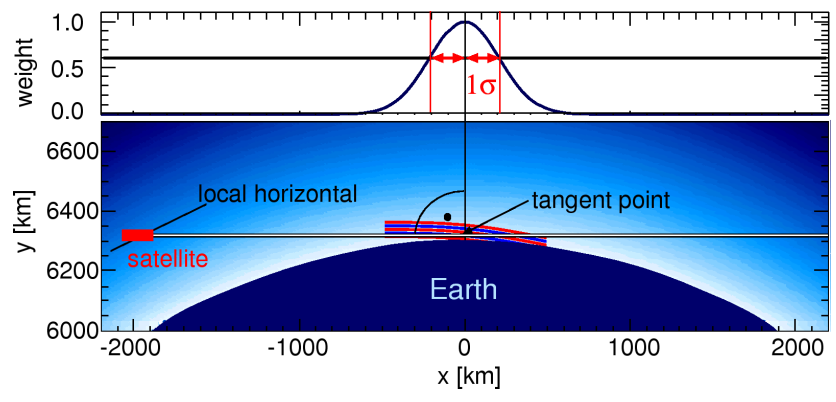

Fig. 2. Viewing geometry of a limb-sounding satellite. The upper panel shows the Gaussian-shaped weighting function along the limb ray. The lower panel indicates sounding of a GW of $400 \mathrm{~km}$ horizontal and $25 \mathrm{~km}$ vertical wavelength. The figure is up to scale. Note that the phase fronts of the GW are curved and coaligned with the limb ray to the left of the tangent point. For discussion see text.

takes a Gaussian shape of $200 \mathrm{~km} 1 \sigma$ width (corresponding to $\sim 500 \mathrm{~km}$ full width at half maximum). The radiance response to the temperature disturbance by a gravity wave can be estimated by convolving the temperature disturbance with the weighting function. Accordingly, a fast alternating temperature disturbance averages out and does not induce a net radiance signal.

Around the location of the tangent point red and blue curves indicate a GW of $25 \mathrm{~km}$ vertical and $400 \mathrm{~km}$ horizontal wavelength. In a geophysical coordinate system using the local vertical as $\mathrm{z}$-axis (the usual coordinate system for analyzing waves) the phase-lines of this wave would be straight lines. However, in an absolute coordinate system, as in this plot, they are curved and partially coaligned with the line-of-sight (LOS). This coalignment is the reason why limb sounders can see GWs with horizontal wavelengths as short as a few hundred kilometers at all (Preusse et al., 2002). In particular, the coalignment is the reason, why the sensitivity limit is at shorter horizontal wavelengths than even sophisticated estimates of the horizontal resolution (von Clarmann et al., 2009) let expect and therefore sensitivity estimates based on the methods described by von Clarmann et al. (2009) have to involve the full 2-D averaging kernel matrix.

By altering the downward viewing angle lines-of-sight with different tangent altitudes are measured and vertical profiles can be obtained. The vertical resolution is very fine and the limiting factors are the geometry, the vertical sampling and field of view. For infrared limb sounders vertical resolutions of about 1.0 to $1.5 \mathrm{~km}$ are technical feasible.

By detailed radiative transfer and retrieval simulations as well as approximate analytical expressions Preusse et al. (2002) deduced the sensitivity of optically-thin limb sounding for GWs. The sensitivity function estimated for the CRISTA instrument is shown in the insert in the upper right corner of Fig. 3a (reproduced by permission of American Geophysical Union from Fig. 6b of Preusse et al., 2002). The shown sensitivity function is based on full radiative-transfer 

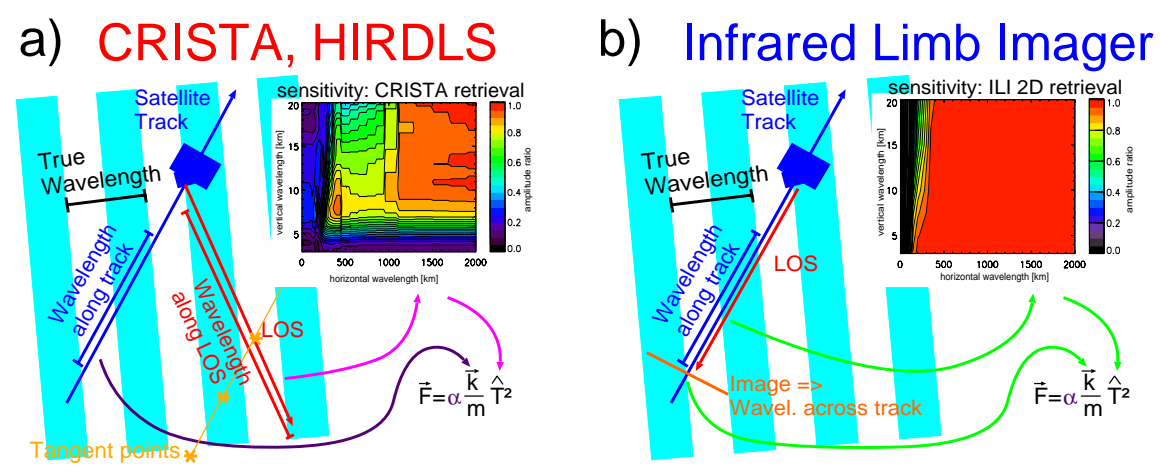

Fig. 3. Schematic drawing comparing GW sounding by (a) current generation satellites as e.g. CRISTA and HIRDLS with (b) a future ILI. The GW wave fronts are indicated by the light-blue shading, the satellite track by the dark-blue arrow, an exemplarily LOS in red, and various tangent points for subsequent profiles in orange. We here have chosen an orientation where wave fronts and satellite-track are oriented differently. The curved arrows from the LOS to the sensitivity function and further to the MF-equation as well as from the satellite track to the MF-equation indicate the main error sources of conventional limb sounders (panel a): degraded sensitivity and missing knowledge on the horizontal wave vector. For an ILI (panel b) backward view and dense along-track sampling result in the correct temperature amplitude; the across-track information by the image is used to determine the full wave vector. A more detailed discussion is given in the text.

and retrieval simulations of monochromatic waves. The horizontal wavelength given is the wavelength projected on the line of sight $\lambda_{h, S}$, which is equal to or longer than the true atmospheric wavelength $\lambda_{h, \mathrm{GW}}$ of the GW. For approximately plane wave fronts (as in Fig. 3) they are related by the angle $\alpha$ between horizontal wave vector and LOS:

$\lambda_{h, S}=\lambda_{h, \mathrm{GW}} \cos (\alpha)$

At short vertical wavelengths the sensitivity is limited by the vertical sampling of the CRISTA measurements, whereas at short horizontal wavelengths the sensitivity is limited by the radiative transfer along the limb ray. While the first can be improved by new instruments, the latter is inherent to the method.

Largest errors are caused for GWs with mid horizontal and vertical wavelengths, e.g. horizontal wavelengths $\lambda_{\text {LOS }}=500 \mathrm{~km}$ along the line-of-sight and vertical wavelengths of $\lambda_{z}=15 \mathrm{~km}$, respectively. Such waves also have a large potential for carrying momentum. For these wavelengths the limb sounder can detect the GW, but with a sensitivity notably smaller than 1 , i.e., the true amplitude is underestimated. If this underestimation cannot be corrected, the induced error for momentum flux can be a factor of two or more, since momentum flux depends on the square of the temperature amplitude (cf. Eq. 2).

The sensitivity discussion indicates: The most pressing problem for estimating momentum flux from current-day satellite measurements is that the input parameters to Eq. (2) are not well known. This is further illustrated by Fig. 3 . The upper panel shows a typical GW observation by a conventional limb sounder as, e.g., CRISTA or HIRDLS. The light-blue shading in the background indicates the GW wave fronts, and the wavelength of this wave is indicated by the black bar. The satellite track from south-west to north-east is given by the blue arrow. In our picture the satellite (in blue) views to the rear and to the left, as is indicated by the line-of-sight (LOS, red arrow). The measurement location is associated with the tangent point (orange asterisk), which is about $2000 \mathrm{~km}$ apart from the satellite. Note that the horizontal wave vector (oriented along the black bar), the satellite track, and the LOS all point into different azimuths in our example. Of course, we had to choose some orientation of the wave fronts and the satellite track for this sketch. In principle, all relative orientations can occur in reality. However, the orientation shown is a rather typical one since preferentially zonal winds frequently result in a preferential south-to-north alignment of the wave fronts.

In order to calculate GW momentum flux the full wave vector and the temperature amplitude must be known. The vertical wavelength can be determined from the vertical profiles with good accuracy. More problematic is the horizontal wave structure. Provided the profiles were sufficiently dense sampled as the satellite moves on, one could infer the horizontal wavelength along the track of the tangent points, which is about the wavelength along the satellite track.

The sampling of the CRISTA instrument was $200 \mathrm{~km}$ along track, and the along-track sampling of HIRDLS was about $100 \mathrm{~km}$. The sampling of these instruments is coarse compared to the visibility filter of IR limb sounding. The observational filter for CRISTA is inserted in the upper right of Fig. 3a, which shows that horizontal wavelengths as short as $200 \mathrm{~km}$ along the line-of-sight can be measured. From this fact alone, aliasing effects due to undersampling of short horizontal wavelength waves can be expected. In addition, the wavelength along the LOS in our example (Fig. 3) is longer than the wavelength along the satellite track. This 


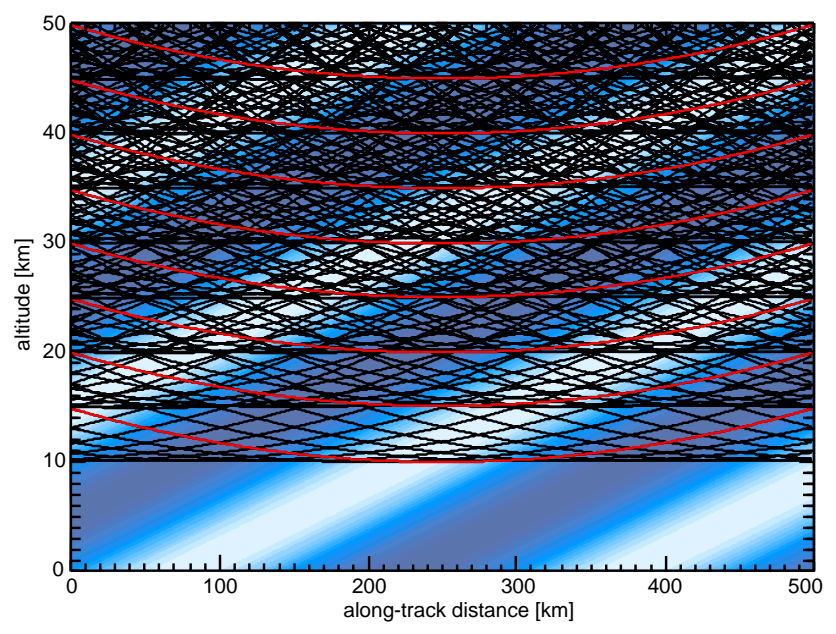

Fig. 4. Multiple limb rays for an along-track sampling of $50 \mathrm{~km}$ and vertical sampling of $5 \mathrm{~km}$ (i.e. 10 times less than envisaged for an ILI) projected onto a GW of $300 \mathrm{~km}$ horizontal and $20 \mathrm{~km}$ vertical wavelength. For details see text.

a) increases the aliasing problem and b) means that we cannot correct for the reduced sensitivity. The latter problem is schematically indicated in Fig. 3a by the pink, curved arrows pointing from the wavelength along track to the sensitivity function and then to the temperature amplitude $\hat{T}$. In addition, we cannot infer from such data the horizontal wave vector $\boldsymbol{k}$ but only the absolute wave number $\left|k_{\text {track }}\right|$ along the satellite track. This value is, in general, lower than the true wavenumber $k$ of the wave, i.e., we can estimate only the absolute value of the momentum flux, and this value will be in general an underestimate. This problem is indicated by the dark purple arrow pointing to $\boldsymbol{k}$. A quantitative assessment of these errors was given by Ern et al. (2004).

Figure $3 \mathrm{~b}$ illustrates how these problems can be overcome by infrared limb imaging. First, the LOS is now coaligned with the satellite track. By using two-dimensional (tomographic) retrievals artifacts caused by the sensitivity function can be largely avoided. The observational filter for a 2-D retrieval is inserted in the upper right of Fig. 3b (details of the retrieval are given in Sect. 2.2). As for the 1-D retrievals, only GWs with horizontal wavelengths longer than about $200 \mathrm{~km}$ are detectable. However, inside the range of GWs detectable by infrared limb sounding the sensitivity is 1, i.e. the amplitude is not degraded by the radiative transfer and retrieval. This is schematically indicated by the green arrows pointing to the sensitivity function and to the temperature amplitude. We will further discuss the benefits of 2-D retrievals in Sect. 2.2. Second, imaging the dimension across-track, the true horizontal wave vector can be determined. This benefit is indicated by the green arrow pointing towards $\boldsymbol{k}$. Imaging of the wave fronts will be demonstrated in Sect. 3. Provided that the sampling along and across track is optimized in compliance with the sensitivity function, all main error sources for deducing GW momentum flux from current-day satellite instruments (cf. Ern et al., 2004) can be eliminated.

\subsection{Two-dimensional retrievals}

As explained in Sect. 2.1, the errors of GW momentum flux crucially depend on the accuracy of the retrieved temperature amplitudes. Preusse et al. (2002) showed that the temperature amplitude is, in general, degraded in conventional retrievals, which assume spherical symmetry and neglect the horizontal structure of the GWs. However, for a backward viewing instrument with sufficient dense sampling tomographic methods can be used (Steck et al., 2005; Carlotti et al., 2006). Figure 4 shows for a $50 \mathrm{~km}$ along-track and a $5 \mathrm{~km}$ vertical sampling distance limb-rays (the lines-of-sight that would be obtained during the measurement) overplotted on a schematic drawing of a GW with $300 \mathrm{~km}$ horizontal and $20 \mathrm{~km}$ vertical wavelength. For Fig. 4 we use a geophysical coordinate system. In contrast to Fig. 2, the phase fronts are straight lines and the limb rays take the shape of parabola.

Note that for a limb imager a vertical sampling of $500 \mathrm{~m}$ is envisaged (Riese et al., 2005; Friedl-Vallon et al., 2006) whereas we used a $5 \mathrm{~km}$ vertical sampling for Fig. 4 , i.e. in order to keep the single rays resolvable in Fig. 4, we reduced the number of limb rays by a factor of 10 . Still we see that there are multiple intersections between the rays. This calls for a two-dimensional, tomographic retrieval which inverts a whole orbit segment at once. Based on the Juelich rapid spectral simulation code (JURASSIC) (Hoffmann, 2006; Hoffmann et al., 2008) such a retrieval was implemented.

The tomographic retrievals discussed in this study are based on the standard optimal estimation approach (Rodgers, 2000), providing the maximum-a-posteriori solution of the inverse problem. A 2-D grid in the line-of-sight plane with $50 \mathrm{~km} \times 1 \mathrm{~km}$ (horizontal $\times$ vertikal) sampling and bilinear interpolations were chosen to represent the atmospheric state. The measurement vector consists of 12.6 micron radiances on a $50 \mathrm{~km} \times 500 \mathrm{~m}$ tangent point grid. The measurement error convariance takes into account noise $\left(0.6 \mathrm{nW} /\left(\mathrm{m}^{2} \mathrm{srcm}^{-1}\right)\right)$ as well as forward model errors $(0.3 \%$, spatially uncorrelated). A first-order autoregressive model is applied to determine the a priori covariance $(20 \mathrm{~K}$ standard deviation, $2 \mathrm{~km}$ vertical correlation length, $500 \mathrm{~km}$ horizontal correlation length).

The code was tested on simulated measurements based on European Centre for Medium-Range Weather Forecasts(ECMWF) data with $0.25^{\circ}$ horizontal resolution. Comparing GW structures visible in ECMWF temperatures with different kind of satellite data (e.g., Wu and Zhang, 2004; Wu and Eckermann, 2008; Schroeder et al., 2009) several studies have shown that ECMWF analyses contain realistic mountain wave features. 

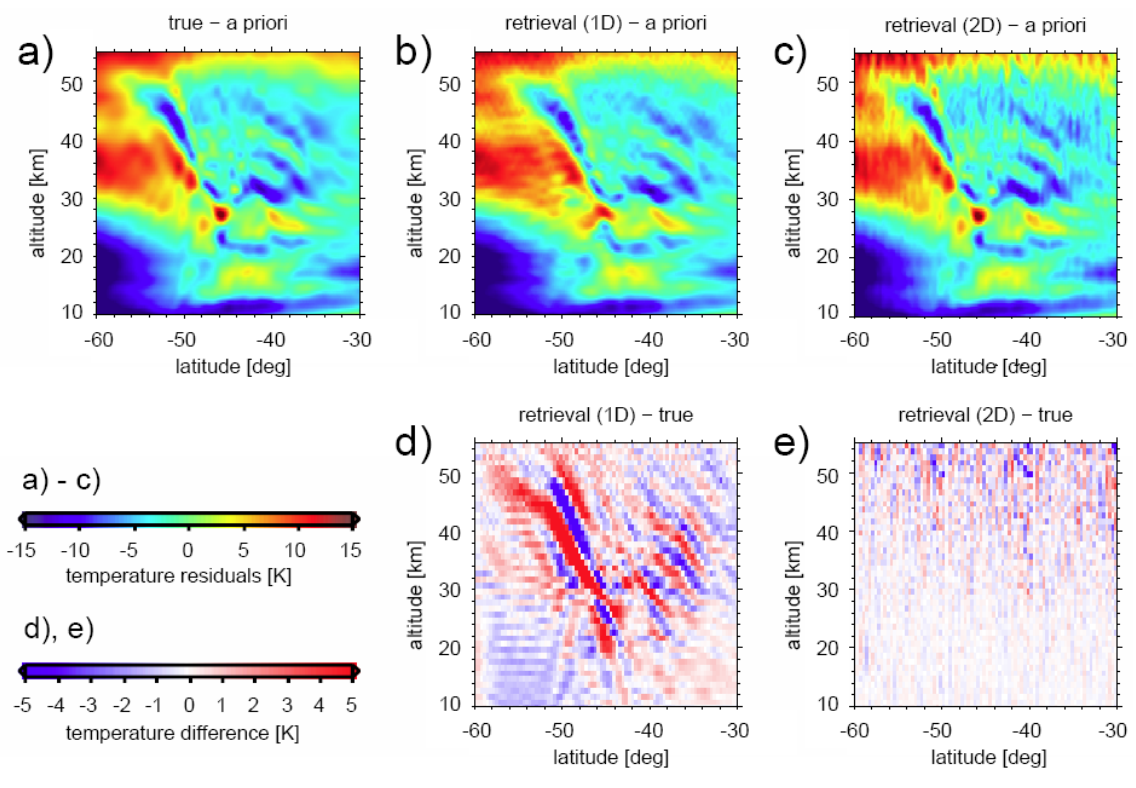

Fig. 5. Gravity wave structures in ECMWF (a) sampled with ILI geometry, and from a full simulation by (b) conventional, one-dimensional and (c) two-dimensional retrieval. The differences in the lower row highlight that for (e) two-dimensional retrieval only noise is seen, but that for (d) conventional, one-dimensional retrieval severe phase shifts are induced. For discussion see text.

In the current study we assume an ILI with 14 horizontal viewing directions. The tangent points of horizontally neighbored viewing directions are apart by an across-track distance of about $24 \mathrm{~km}$ at their tangent points. In addition, we assume a horizontal sampling distance of $50 \mathrm{~km}$ along the satellite track and a vertical sampling distance of $500 \mathrm{~m}$. Simulated measurements for one of the 14 simultaneously measured tracks are shown in Fig. 5. The upper row shows temperature fields detrended by one representative profile. For panel (a) the ECMWF temperatures were simply sampled at the positions of the tangent points. This serves as a reference of the true temperature distributions. In panels (b) and (c) full simulations of the measurements including radiative transfer, instrument parameters (e.g. noise), and retrieval are shown. The lower row shows the differences between the respective retrieval simulation and the reference. Twodimensional retrievals (panel c) can reliably recover the atmospheric structures and, accordingly, the differences given in panel (e) show only noise.

For conventional, one-dimensional retrievals shown in panel (b) this is different. Smaller structures as e.g. the warm spot at $47^{\circ} \mathrm{S}, 29 \mathrm{~km}$ altitude are smeared out and the tilted GW phase fronts are shifted in phase. This phase shift is particularly emphasized in the differences in panel (d). Such phase shifts are expected from GW sounding theory (cf. Fig. 8 of Preusse et al., 2002) since the wave signal stems from the leg of the parabola where the GW phases are coaligned with the LOS, i.e. the phases do not correspond to the tangent point, but are shifted to one side of the parabola.
This has very important implications for the interpretation of the measurements. Gravity wave structures deduced from two-dimensional retrievals can be directly compared to e.g. high resolution modeling since they represent the correct phase structures. Gravity wave structures deduced from conventional, one-dimensional retrievals display phase shifts. If the GW is viewed by one instrument at a constant viewing angle, they still can be used for inference of GW momentum flux, since this requires only horizontal phase-differences, not the absolute phase. However, if several instruments have to be combined and the horizontal orientation of the LOS changes, the induced phase shifts can vary and deduced horizontal wavelengths and GW momentum flux values are, in general, not reliable. The latter for instance might happen, if several GPS radio occultation sensors were combined to obtain sufficient dense sampling for GW momentum flux inference.

\section{Measurement-modeling comparison and gravity wave source identification}

Precise characterizations of GWs by an ILI will benefit GW research in three ways. First, global climatologies of GW momentum flux can be used for constraining global GW modeling, for instance to tune the free parameters of a $\mathrm{GW}$ parameterization scheme; second, direct comparisons between measured and modeled temperature structures can be used to validate e.g. mesoscale modeling; and third, retrieved GW parameters can be used to test concepts of wave propagation and infer GW sources. 


\subsection{Global constraints}

For absolute values of momentum flux deduced from CRISTA a very conservative error analysis indicates an error range of about a factor of 5 (Ern et al., 2004), a more realistic error estimate results in about a factor of 2 . However, despite these large errors CRISTA GW momentum flux estimates still can be employed to constrain global GW modeling (Ern et al., 2004; Ern et al., 2006; Preusse et al., 2009). In addition, GW momentum flux estimates from CRISTA-1 were validated against balloon measurements and agree much better than the error limits would let expect (Hertzog et al., 2008). Using limb-imaging technique GW momentum flux could be deduced with largely improved accuracy by eliminating all leading error sources listed in Ern et al. (2004) (cf. Sect. 2.1) and would hence result in much more stringent constraints for global GW modeling, including constraints on GW parameterization schemes such as designed by Medvedev and Klaassen (1995); Hines (1997); Alexander and Dunkerton (1999) or Warner and McIntyre (2001), and GW source parameterizations for, e.g., mountain waves (McFarlane, 1987), frontal systems (Charron and Manzini, 2002) and convection (Beres et al., 2004; Song and Chun, 2005).

In addition to increased accuracy an ILI can improve a second issue of current GW climatologies. Analyzing undersampled data sets from CRISTA or HIRDLS, one needs to focus on the leading structure in terms of temperature amplitude. However, these largest amplitude GWs are not necessary the only important ones. High phase speed GWs that have still small amplitudes in the lower stratosphere (the observation altitude) might become important at higher altitudes. The much denser sampling of an ILI provides far more information on the larger amplitude structures. They hence can be determined with higher accuracy and this should allow to subtract them and search for smaller amplitudes. This would be a technique similar to the one we employ to isolate GWs from planetary waves by subtracting zonal wavenumbers 0-6.

Two examples where this could prove to be important are the tropics and the winter polar jets. The tropical GW fields are dominated by large scale GWs of several $1000 \mathrm{~km}$ horizontal wavelengths. These waves carry very small momentum fluxes and, accordingly, a tropical maximum in GW potential energy becomes a minimum of tropical GW momentum flux when evaluating the CRISTA and HIRDLS data (Ern et al., 2004; Alexander et al., 2008b). However, it strikes odd that there should be no smaller scale GWs connected to the rain bands of the Intertropical Convergence Zone (ITCZ). Are they really not present or just overlooked when analyzing the large amplitude, large horizontal wavelength waves in the tropics? An ILI could answer this question. Similarly, in the strong polar jets at the edges of the winter polar vortices the dominant GWs propagate westward, i.e. in opposite direction to the wind velocity. However, the wind reversal

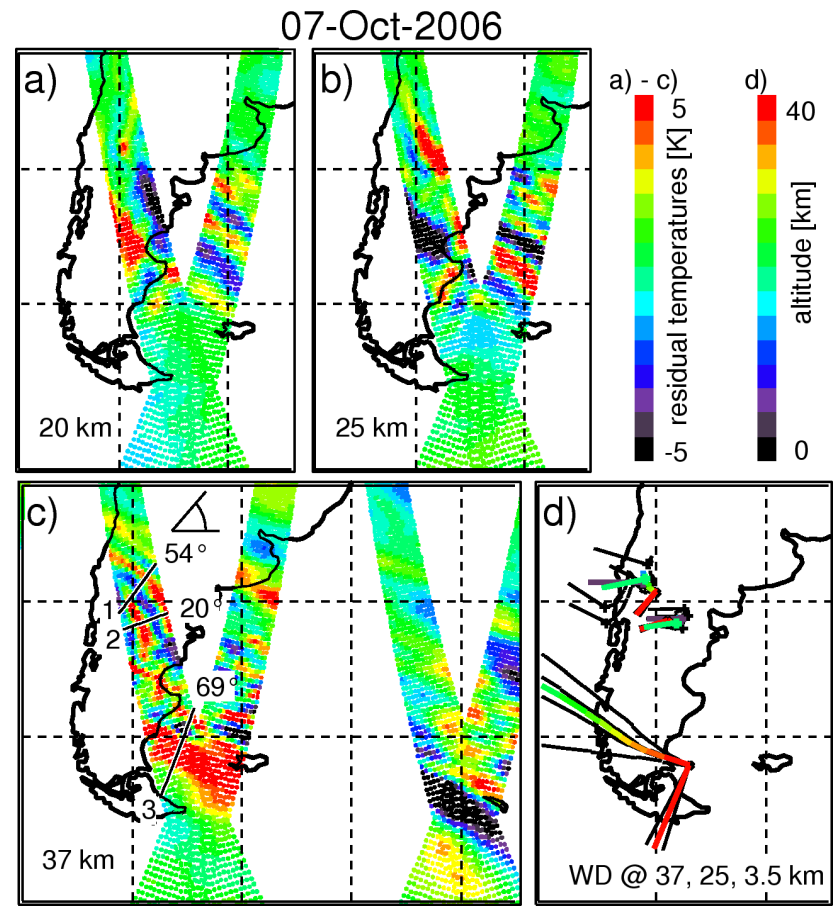

Fig. 6. ECMWF temperatures detrended by wavenumber 0-6 sampled on an ILI measurement grid for altitudes of $20 \mathrm{~km}$ (a), $25 \mathrm{~km}$ (b) and $37 \mathrm{~km}$ (c). At $37 \mathrm{~km}$ for three cases the GW propagation direction was determined and sections along these lines labeled 1, 2, and 3 are indicated by black lines. Wave parameters determined from these sections (see also Fig. 7) serve as input for back-tracing of the GWs to their source altitude. The results are shown in (d). For details see text.

at the mesopause can be caused only by eastward propagating GWs. Again, an ILI will help to identify these weaker components.

\subsection{Mesoscale modeling and GW resolving global modeling}

Gravity wave measurements from an ILI would be ideally suited for model validation. A first model comparison was performed by Preusse et al. (2002) (cf. Fig. 16) for a case study of mountain waves over the southern Andes. The MM5 model was able to reproduce the amplitudes as well as the phase structure of mountain waves measured by CRISTA above Tierra del Fuego. More detailed comparisons were performed for instance by Eckermann et al. (2006) validating a variety of numerical models by nadir viewing data from the Advanced Microwave Sounding Unit (AMSU). The capability of the satellite instrument to produce horizontal maps provides a view on the wave which is very similar to the model results. This is a large improvement regarding the localized profiles provided by e.g. radiosondes. There is a second advantage of the satellite data. Recent work by Kim et al. (2008) uses the AMSU data to validate GWs simulated 


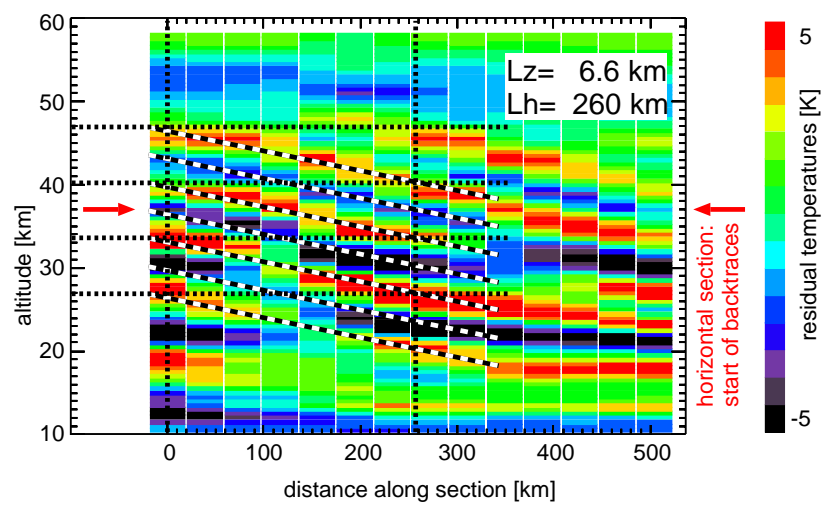

Fig. 7. Vertical section along the black line labeled "1" in Fig. 6c. From this section horizontal and vertical wavelength of the dominant GW can be determined and used as input parameter for backward ray tracing studies (see also Fig. 6). For details see text.

above a typhoon. Such severe weather events are an important source of GWs but prevent measurements by in-situ techniques.

However, nadir viewing instruments are sensitive to long vertical wavelength GWs (larger than about $12-15 \mathrm{~km}$ ), and in the stratosphere only. This is a serious limitation strongly restricting the number of cases, since in the stratosphere such events only occur in exceptionally high background winds or for very high phase-speed GWs and are therefore rare. The ILI, however, can view also the short vertical wavelength waves and thus the bulk of those events. It should be noted that in the cases described here the horizontal wavelength would be sufficiently long to be also visible by a limb sounder.

Recently also global models like ECMWF and the KANTO GCM (Watanabe et al., 2008) have reached a spatial resolution allowing them to explicitly resolve parts of the GW spectrum. These global models are computationally much too expensive to be used for climate prediction runs, but based on case studies they can provide valuable information on the interaction of GWs with the background winds without such tedious assumptions as purely vertical and instantaneous GW propagation or neglect of wave-wave interactions. Shortest horizontal scales properly resolved by global GW resolving models are GWs with horizontal wavelengths of $200-300 \mathrm{~km}$. However, even if the waves are resolved, the wave sources invoke much smaller scales and the global models must be validated (cf. Preusse et al., 2008). Some first intercomparisons have been performed, but they are either limited on case studies (Eckermann et al., 2006) or, though globally, they are restricted on comparing temperature amplitudes (Schroeder et al., 2009). An ILI can provide global and detailed information on the full range of GWs in GW resolving GCMs.

\subsection{Source backtracing}

Nadir viewing instruments provide the horizontal propagation direction of GWs. However, their limited vertical resolution $\left(\lambda_{z}>15 \mathrm{~km}\right)$ combined with their limited vertical coverage (25-40 km altitude) normally does not allow a full characterization of the wave, since the vertical wavelength remains unknown. A full wave characterization, however, can lead to new insights in GW sources and GW propagation:

Figure 6 shows ECMWF temperatures detrended by wavenumber 0-6 sampled on an ILI measurement grid for altitudes of $20 \mathrm{~km}$ (panel a), $25 \mathrm{~km}$ (panel b) and $37 \mathrm{~km}$ (panel c). At all altitudes we observe wavelike temperature structures. At $37 \mathrm{~km}$ we have identified for three cases the propagation direction (i.e., the direction rectangular to the wave fronts) and made vertical cross sections along these lines. An example is given in Fig. 7 which shows the vertical cross section along the wave propagation direction of the northernmost of the three wave events (cf. the line labeled "1" in Fig. 6c). From this section we can infer the horizontal and vertical wavelength. Using also background winds from ECMWF, we now can fully characterize the wave and perform backtracing calculations shown in Fig. $6 \mathrm{~d}$. The red, green and dark purple bars give the propagation direction and horizontal wavelength at $37 \mathrm{~km}, 25 \mathrm{~km}$ and $3.5 \mathrm{~km}$ altitude based on best estimates of the wave parameters for the three examples. The color code of multi-colored traces gives the altitude. Black traces show variations of \pm 5 degree in launch direction and $\pm 0.5 \mathrm{~km}$ in launch vertical wavelength, which are realistic error ranges.

For the first case (case 1) we see that the wave is launched with a south-west orientation but turns to a westward orientation as we back-trace it to lower altitudes. Simultaneously the horizontal wavelength becomes longer. This is exactly what we observe also in Fig. 6b. At the source level $(3.5 \mathrm{~km}$ altitude) three of the backtraces are over the Andes, two very close to coast over the sea. This together with the westward orientation of the wave vectors (rectangular to the Andes and along the main wind direction) is an indication for a mountain wave. Even more clearly the second case can be backtraced to orography and has a westward wave propagation direction at source level. The third wave case is different. Here we observe a wave of unclear origin, which is definitely not a mountain wave. The obtained source position close to the vortex edge (cf. also the temperature structure in Fig. 5) suggests that this wave is generated, indeed, at this location, however not by the orography but by strong wind shear or geostrophic adjustment.

The discussed example shows that interpreting the observed wave structures as monochromatic waves results in physically reasonable interpretations. When an ILI really will be flown in space, new custom-tailored analysis methods will be generated for, e.g., analyzing spectra in along-track and across-track direction, for fitting multiple waves and for automatic detection of the wave parameters. 


\section{Assessment of different techniques to measure momentum flux}

We have discussed so far the value of an ILI for GW research in its own right. However, satellites are immensely expensive research platforms and should therefore primarily be used to gain information which is not easily accessible from other measurement techniques. One immediate advantage of satellite remote sensing is true global coverage at a reasonable temporal resolution. A second advantage is that we can obtain truly three-dimensional information on GWs in a wide wavelength range. There are, however, also limitations. For instance, gravity waves with horizontal wavelengths shorter than about $100 \mathrm{~km}$ cannot be assessed. Gravity waves with horizontal wavelengths between $30 \mathrm{~km}$ and $100 \mathrm{~km}$ could potentially be important for conveying momentum from the troposphere to the mesosphere. These scales are very difficult to address from space and due to a lack of global observations their true importance is unknown (Preusse et al., 2008). However, we know from CRISTA and SABER observations that the momentum carried by the waves visible to infrared limb-sounding is considerable and might alone suffice to drive the winds in the mesosphere (Preusse et al., 2006, 2009). A second issue already raised before is that we cannot directly measure GW momentum flux, i.e., $\left(u^{\prime} w^{\prime}, v^{\prime} w^{\prime}\right)$, from space. Therefore we have to infer GW momentum flux from temperature perturbations invoking GW polarization relations. How do such restrictions compare to other techniques measuring GWs? How important is the new information for GW research? A true assessment of the value of an ILI requires to compare the ILI measurements to other measurement techniques.

On global scale the most important impact of GWs is to couple different layers of the atmosphere by conveying momentum and driving atmospheric wind systems. Thus, true constraints as required e.g. for general circulation modeling can be deduced only from global datasets of GW momentum flux including a large part of the GW spectrum and covering a wider range of altitudes. At least the seasonal cycle and the QBO should be resolvable. Assessing different measurement techniques we should therefore consider the following criteria:

\section{- Direct GW momentum flux measurements}

Direct measurements of $\left(u^{\prime} w^{\prime}, v^{\prime} w^{\prime}\right)$ allow for direct inference of GW momentum flux. In this way one has not to rely on dispersion and polarization relations.

\section{- GW momentum flux measurements}

Though there is a number of climatologies based on temperature or wind variances, CRISTA and HIRDLS data show that global distributions of GW momentum flux are very different (Ern et al., 2004; Alexander et al., 2008b). Model results indicate that also the seasonal cycle may be different. It is therefore very important to infer GW momentum flux.
- Spatial coverage

We are most concerned in global effects of GWs, so our data need to be global.

\section{- Altitude coverage}

The shape of the vertical wavenumber spectrum changes with altitude. Waves important in the mesosphere may have very low amplitudes in the lowermost stratosphere. The larger the altitude coverage is, the more valuable is the measurement.

\section{- Spectral coverage}

Most measurement techniques can address only a certain part of the GW spectrum. The larger this part is, the more valuable is the measurement. To assess those waves which can convey momentum from the troposphere to the mesosphere horizontal wavelengths between $30 \mathrm{~km}$ and a few $1000 \mathrm{~km}$ and vertical wavelengths between $3 \mathrm{~km}$ and a few $10 \mathrm{~km}$ should be monitored (Preusse et al., 2008).

\section{- Temporal coverage}

To quantify the effects of e.g. the annual cycle and the QBO one needs to be able to resolve the annual and interannual variations of GW momentum flux. For example, GW momentum flux changes more than one order of magnitude from summer to winter.

Having defined the criteria, we can now survey all techniques used for GW research in the middle atmosphere in Table 1. (For an overview of momentum flux studies from a variety of different instruments see Preusse et al., 2008). It becomes evident even from a first glance that all techniques are far from being perfect.

Our first criterion is to which extent momentum flux measurements and, in particular, direct measurements of the wind fluctuations are possible. Table 1 shows that there are only four techniques measuring GW momentum flux directly, i.e. measuring horizontal and vertical wind perturbations. Research aircrafts, radars and lidars provide data sets so limited that they can be used only for studying some GW related processes but are not of use to constrain global GW modeling at all (keep in mind that only few research aircrafts, selected radars, and the best wind lidars are capable of measuring GW momentum flux).

The second group of criteria aims at the spatial and temporal coverage required to assess the global influence of GWs. Only satellites provide such global data at a reasonable temporal resolution and coverage. However, only infrared limb sounders so far have proven to be able to measure absolute values of GW momentum flux. There is a case-study of a mountain wave above South America (Wu and Eckermann, 2008) showing that amplitude, vertical and along-track horizontal wavelength can also be deduced from AURA-MLS measurements. In future it should therefore also be possible to deduce absolute values of GW momentum flux from these 
Table 1. Comparison of all measurement techniques employed for assessing middle atmosphere GWs. Quantities which can provided or criteria which are matched are marked by "+", such which cannot are marked by "-". For temporal coverage "0" indicates temporally resolved data sets of several months length, but on campaign basis, i.e. no continuous multi-year data available. Particularly critical criteria are highlighted in boldface. For IR limb measurements absolute values of GW momentum flux so far have been derived up to altitudes of $60 \mathrm{~km}$. An extension of this altitude range to altitudes of $90 \mathrm{~km}$ (or even higher) should be possible without major difficulty. For discussion see text.

\begin{tabular}{|c|c|c|c|c|c|c|}
\hline & $u^{\prime} w^{\prime}$ & MFlux & spatial coverage & altitude range & spectral coverage & temporal coverage \\
\hline \multicolumn{7}{|l|}{ In-situ: } \\
\hline radiosonde & - & + & no ocean & $<30 \mathrm{~km}$ & $\lambda_{z}<10 \mathbf{k m}$ & + \\
\hline rocketsonde & - & + & no ocean & $<50 \mathrm{~km}$ & $\lambda_{z}<10 \mathrm{~km}$ & - \\
\hline research aircraft & + & + & few campaigns & ca. $20 \mathrm{~km}$ & $\lambda_{h}<500 \mathrm{~km}$ & - \\
\hline super-pressure balloon & + & + & polar vortices, equator & ca. $20 \mathrm{~km}$ & in future: + & $\mathbf{0}$ \\
\hline \multicolumn{7}{|l|}{ Ground-based: } \\
\hline radar & + & + & order of 10 stations & $<20 ;>70 \mathrm{~km}$ & $\tau<10 \mathrm{~h}$ & 0 \\
\hline lidar & + & + & order of 1 station & $85-100 \mathrm{~km}$ & $\tau<14 \mathrm{~h}$ & good weather \\
\hline \multicolumn{7}{|l|}{ Satellite: } \\
\hline nadir & - & $?$ & global & $20-40 \mathrm{~km}$ & $\lambda_{z}>14 \mathrm{~km}$ & + \\
\hline GPS & - & - & global & $10-30 \mathrm{~km}$ & $\lambda_{h}>100 \mathrm{~km}$ & + \\
\hline microwave sub-limb & - & Abs.? & global & $15-70 \mathrm{~km}$ & $\lambda_{z}>9 \mathrm{~km}$ & + \\
\hline infrared limb & - & Abs. & global & $15-60(90) \mathrm{km}$ & $\lambda_{h}>100 \mathrm{~km}$ & + \\
\hline IR limb imager (ILI) & - & + & global & $15-60(90) \mathrm{km}$ & $\lambda_{h}>100 \mathrm{~km}$ & + \\
\hline
\end{tabular}

microwave measurements. This would nicely complement the CRISTA and HIRDLS results because slightly shorter horizontal scales are measured. A detailed assessment of the benefits of combining several satellite measurements for quantifying most of the spectral range of vertically propagating GWs is given by Preusse et al. (2008). Recently, Horinouchi and Tsuda (2008) made use of a special constellation of the six COSMIC satellites carrying GPS receivers. The COSMIC satellites were launched into the same orbit and several of these satellites remained close together for more than half a year. In total a few thousand profile-coincidences were found and some inferences about the horizontal wave structures could be made, though aliasing effects as found in CRISTA data were also obvious in the presented altitudedistance cross-sections. Though an increasing number of GPS receivers will be available in future, coverage will remain insufficient to deduce GW momentum flux, in particular since the wave must be sounded at the same LOS angle (cf. Sect. 2.2).

In summary, none of the techniques can match all the criteria. There are three techniques, however, which provide detailed information on GWs and their momentum flux at a good spatial and temporal coverage. These three most promising techniques are radiosondes, super-pressure balloons and infrared limb imaging. Compared to both in-situ techniques the ILI can provide a better altitude coverage (up to the stratopause) and true global coverage.

\subsection{Radiosondes}

Radiosondes provide accurate measurements of temperature and horizontal wind with high vertical resolution. However, due to the vertical detrending required to isolate GWs from other atmospheric structures, and due to the limited altitude range, radiosondes are limited in their vertical wavelength range. Although often the peak of the vertical wavenumber spectrum in the lower stratosphere is included, this is a serious limitation for determining the GWs relevant for the mesosphere (Preusse et al., 2008). Dense radiosonde networks are available for the United States of America and Canada, Europe and parts of Asia, and Australia.

\subsection{Super-pressure balloons}

Super-pressure balloons are the only technique measuring directly the wind perturbations and intrinsic wave frequency. The balloons are being continuously improved. So far they have provided GW momentum flux analyses for a frequency range corresponding closely to the wavelength-range of infrared-limb sounding measurements, and the resulting GW momentum flux agrees very well (Hertzog et al., 2008). In future they will also provide information on highfrequency GWs close to the buoyancy frequency. Their main limitations are the (for the stratosphere) low measurement altitude $(<20 \mathrm{~km})$ and their use only in campaigns. In particular, for political and safety reasons the balloons can be flown in a self-contained air-mass, such as a winter or spring polar vortex or in the tropics only. For technical and logistic 
reasons the balloons cannot be launched in mid-winter in Antarctica. This prevents these measurements to be used for whole annual cycle or QBO analyses (confinement in the tropics is found only for QBO east-phase at flight altitude). In addition, even in campaigns the number of balloons is limited and information must be deduced from single balloons. Accordingly, the separation of GWs from the background and their further analysis involves to describe the wind perturbations as a superposition of a few monochromatic waves.

\subsection{Infrared limb imaging}

True progress of GW research on the global scale therefore must involve an ILI which is the sole instrument providing GW momentum flux at good precision with true global coverage throughout the entire seasonal cycle and QBO for a wider altitude range. These measurements can be complemented and validated against in-situ techniques such as radiosondes and super-pressure balloons. Combining the precise characterization of the temperature structure with wind measurements from in-situ or ground-based instruments, reliable tests of the $\mathrm{GW}$ polarization relation relating temperature and wind amplitudes would be possible.

\section{Summary}

In the recent years satellite measurements of atmospheric gravity waves (GWs) largely contributed to scientific progress in the field of GWs. The two most important achievements were images of GWs from nadir viewing satellite instruments and global estimates of GW momentum flux from infrared limb sounders. Temperature signatures of GWs modeled by mesoscale models and mapped by nadir viewers were compared in terms of the extent of the wave field, the amplitudes and the propagation characteristics in a direct and intuitive manner. In addition, satellites can measure above severe weather events such as typhoons inaccessible by in-situ soundings. Global fields of absolute values of GW momentum flux were used to constrain global GW models including GW parameterization schemes used in chemistryclimate modeling. The logical next step is to combine the advantages of the two techniques, i.e., the good vertical resolution of infrared limb sounding and the horizontal mapping capabilities of the nadir viewing satellites and generate truly three-dimensional data fields of GWs. This can be achieved by an infrared limb imager (ILI). The observational filter of this measurement technique is sufficiently wide in vertical wavelength to cover the entire range of vertically propagating GWs. In terms of horizontal wavelength a large and important part of the wave spectrum is resolved.

Infrared limb imaging of GWs opens many new opportunities for GW research. The regular sampling grid can be used for simultaneous spectral analysis of vertical and horizontal GW scales. In a multi-wave approach weaker GW signatures can be isolated in the presence of dominating components. Such approaches could be very important, since weaker GW components may carry as much momentum as GWs having large amplitudes but at the same time large horizontal wavelengths. This could be especially the case in the tropics. In addition, eastward propagating GWs with unfavorable propagation directions in the strong westerlies of the stratospheric winter polar vortices are required for the wind reversal at the mesopause. Furthermore, full wave characterization from the 3-D wave structures can be used for testing wave propagation concepts and also to localize and characterize GW sources by e.g. GW backtracing.

For all these kind of case-study like approaches an ILI provides novel information which in this form cannot be provided by any other measurement technique. If the full wave characterization from the temperature structures is compared to direct measurements of the GW induced wind perturbations $\left(u^{\prime} w^{\prime}, v^{\prime} w^{\prime}\right)$ measured by super-pressure balloons or radars, GW polarization relations can be tested. In fact, absolute values of GW momentum flux from CRISTA well agree with in-situ measurements by super-pressure balloons.

In an assessment of all measurement techniques, an ILI compares very favorable even for process studies. Statistically valuable information can be gained by radiosondes, super-pressure balloons and satellite instruments. However, only an ILI can provide reliable global estimates of GW momentum flux for a wider altitude region over the whole seasonal cycle required to assess the global effects of GWs.

Acknowledgements. We thank the European Centre for MediumRange Weather Forecasts (ECMWF) for providing the highresolution reanalysis data used in our simulations. We thank Fernand Karcher and one anonymous reviewer as well as Thomas von Clarmann for their comments which helped to clarify some points in the manuscript. The sensitivity function shown in Fig. 2 is reproduced by permission of American Geophysical Union from Fig. 6b of Preusse et al. (2002), Copyright 2002 American Geophysical Union.

Edited by: J.-L. Attie

\section{References}

Adams, S., Spang, R., Preusse, P., and Heinemann, G.: The benefit of limb cloud imaging for tropospheric infrared limb sounding, Atmos. Meas. Tech., 2, 287-298, 2009,

http://www.atmos-meas-tech.net/2/287/2009/.

Alexander, M. J. and Dunkerton, T. J.: A spectral parameterization of mean-flow forcing due to breaking gravity waves, J. Atmos. Sci., 56, 4167-4182, 1999.

Alexander, M. J. and Rosenlof, K. H.: Gravity-wave forcing in the stratosphere: Observational constraints from the Upper Atmosphere Research Satellite and implications for parameterization in global models, J. Geophys. Res., 108, 4597, doi:1029/2003JD003373, 2003. 
Alexander, M. J., Teitelbaum, H., Eckermann, S. D., Gille, J., Barnett, J., and Barnet, C.: High-Resolution Satellite Observations of Mountain Waves, B. Am. Meteorol. Soc., 89(2), 151-152, 2008a.

Alexander, M. J., Gille, J., Cavanaugh, C., and 15 further coauthors: Global estimates of gravity wave momentum flux from High Resolution Dynamics Limb Sounder observations, J. Geophys. Res., 113, D15S18, doi:10.1029/2007JD008807, 2008b.

Beres, J. H., Garcia, R. R., Boville, B. A., and Sassi, F.: Implementation of a gravity wave source spectrum parameterization dependent on the properties of convection in the Whole Atmosphere Community Climate Model (WACCM), J. Geophys. Res., 110, D10108, doi:10.1029/2004JD005504, 2005.

Carlotti, M., Brizzi, G., Papandrea, E., Prevedelli, M., Ridolfi, M., Dinelli, B. M., and Magnani, L.: GMTR: Two-dimensional geofit multitarget retrieval model for Michelson interferometer for passive atmospheric sounding/environmental satellite observations, Appl. Optics, 45, 716-727, 2006.

Charron, M. and Manzini, E.: Gravity waves from fronts: Parameterization and middle atmosphere response in a general circulation model, J. Atmos. Sci., 59, 923-941, 2002.

Dunkerton, T. J.: The role of gravity waves in the quasi-biennial oscillation, J. Geophys. Res., 102, 26053-26076, 1997.

Eckermann, S. D., Wu, D. L., Doyle, J. D., Burris, J. F., McGee, T. J., Hostetler, C. A., Coy, L., Lawrence, B. N., Stephens, A., McCormack, J. P., and Hogan, T. F.: Imaging gravity waves in lower stratospheric AMSU-A radiances, Part 2: Validation case study, Atmos. Chem. Phys., 6, 3343-3362, 2006,

http://www.atmos-chem-phys.net/6/3343/2006/.

Ern, M., Preusse, P., Alexander, M. J., and Warner, C. D.: Absolute values of gravity wave momentum flux derived from satellite data, J. Geophys. Res., 109, D20103, doi:10.1029/2004JD004752, 2004.

Ern, M., Preusse, P., and Warner, C. D.: Some experimental constraints for spectral parameters used in the Warner and McIntyre gravity wave parameterization scheme, Atmos. Chem. Phys., 6, 4361-4381, 2006,

http://www.atmos-chem-phys.net/6/4361/2006/.

Friedl-Vallon, F., Riese, M., Maucher, G., Lengel, A., Hase, F., Preusse, P., and Spang, R.: Instrument concept and preliminary performance analysis of GLORIA, Adv. Space Res., 37, 22872291, doi:10.1016/j.asr.2005.07.075, 2006.

Fritts, D. C. and Alexander, M. J.: Gravity wave dynamics and effects in the middle atmosphere, Rev. Geophys., 41(1), 1003, doi:10.1029/2001RG000106, 2003.

Hertzog, A., Boccara, G., Vincent, R. A., Vial, F., and Cocquerez, P.: Estimation of gravity wave momentum flux and phase speeds from quasi-Lagrangian stratospheric balloon flights. Part II: Results from the Vorcore campaign in Antarctica, J. Atmos. Sci., 65, 3056-3070, 2008.

Hines, C. O.: Doppler-spread parameterization of gravity-wave momentum deposition in the middle atmosphere. Part 1: Basic formulation, J. Atmos. Sol.-Terr. Phys., 59, 371-386, 1997.

Hoffmann, L.: Schnelle Spurengasretrieval für das Satelliteninstrument Envisat MIPAS, Tech. Rep. JUEL-4207, Forschungszentrum Jülich, Germany, ISSN 0944-2952, 2006.

Hoffmann, L., Kaufmann, M., Spang, R., Müller, R., Remedios, J. J., Moore, D. P., Volk, C. M., von Clarmann, T., and Riese, M.: Envisat MIPAS measurements of CFC-11: retrieval, validation, and climatology, Atmos. Chem. Phys., 8, 3671-3688, 2008, http://www.atmos-chem-phys.net/8/3671/2008/.

Horinouchi, T. and Tsuda, T.: Spatial structures of stratospheric gravity waves derived from COSMIC GPS occultation data, SPARC 4th General Assembly, Bologna, Italy, 31 August-6 September 2008, abstract no. 00412, 2008.

Kim, S.-Y., Chun, H.-Y., and Wu, D. L.: A study on gravity waves generated by typhoon Ewiniar using mesoscale model and observations, EGU General Assembly, Vienna, Austria, 13-18 April 2008, EGU2008-A-07316, 2008.

Mayr, H. G., Mengel, J. G., Wolff, C. L., and Porter, H. S.: QBO as potential amplifier of solar cycle influence, Geophys. Res. Lett., 33, L05812, doi:10.1029/2005GL025650, 2006.

Mayr, H. G., Mengel, J. G., Wolff, C. L., Huang, F. T., and Porter, H. S.: The QBO as potential amplifier and conduit to lower altitudes of solar cycle influence, Ann. Geophys., 25, 1071-1092, 2007, http://www.ann-geophys.net/25/1071/2007/.

McFarlane, N. A.: The effect of orographically excited gravity wave drag on the general circulation of the lower stratosphere and troposphere, J. Atmos. Sci., 44, 1775-1800, 1987.

McLandress, C.: On the importance of gravity waves in the middle atmosphere and their parameterization in general circulation models, J. Atmos. Sol.-Terr. Phys., 60, 1357-1383, 1998.

McLandress, C. and Shepherd, T. G.: Simulated anthropogenic changes in the Brewer-Dobson circulation, including its extension to high altitudes, J. Climate, 22, 1516-1540, 2008.

Medvedev, A. S. and Klaassen, G. P.: Vertical evolution of gravity wave spectra and the parameterization of associated wave drag, J. Geophys. Res., 100, 25841-25853, 1995.

Preusse, P., Dörnbrack, A., Eckermann, S. D., Riese, M., Schaeler, B., Bacmeister, J. T., Broutman, D., and Grossmann, K. U.: Space based measurements of stratospheric mountain waves by CRISTA, 1. Sensitivity, analysis method and a case study, J. Geophys. Res., 107, 8178, doi:10.1029/2001JD000699, 2002.

Preusse, P., Ern, M., Eckermann, S. D., Warner, C. D., Picard, R. H., Knieling, P., Krebsbach, M., Russell III, J. M., Mlynczak, M. G., Mertens, C. J., and Riese, M.: Tropopause to mesopause gravity waves in August: Measurement and modeling, J. Atmos. Sol.-Terr. Phys., 68, 1730-1751, 2006.

Preusse, P., Eckermann, S. D., and Ern, M.: Transparency of the atmosphere to short horizontal wavelength gravity waves, J. Geophys. Res., 113, D24104, doi:10.1029/2007JD009682, 2008.

Preusse, P., Eckermann, S. D., Ern, M., Oberheide, J., Picard, R. H., Roble, R. G., Riese, M., Russell III, J. M., and Mlynczak, M. G.: Global ray tracing simulations of the SABER gravity wave climatology, J. Geophys. Res., 114, D08126, doi:10.1029/2008JD011214, 2009.

Riese, M., Friedl-Vallon, F., Spang, R., Preusse, P., Schiller, C., Hoffmann, L., Konopka, P., Oelhaf, H., von Clarmann, T., and Höpfner, M.: GLObal limb Radiance Imager for the Atmosphere (GLORIA): scientific objectives, Adv. Space Res., 36, 989-995, doi:10.1016/j.asr.2005.04.115, 2005.

Rodgers, C. D.: Inverse Methods for Atmospheric Sounding: Theory and Practice, Series on Atmospheric, Oceanic and Planetary Physics, World Scientific, 2000.

Schroeder, S., Preusse, P., Ern, M., and Riese, M.: Gravity waves resolved in ECMWF and measured by SABER, Geophys. Res. Lett., 36, L10805, doi:10.1029/2008GL037054, 2009.

Shuckburgh, E., Norton, W., Iwi, A., and Haynes, P.: Influence 
of the quasi-biennial oscillation on isentropic transport and mixing in the tropics and subtropics, J. Geophys. Res., 106, 1432714337, 2001.

Sigmond, M., Kushner, P. J., and Scinocca, J. F.: Discriminating robust and non-robust atmospheric circulation responses to global warming, J. Geophys. Res., 112, D20121, doi:10.1029/2006JD008270, 2007.

Sigmond, M., Scinocca J. F., and Kushner, P. J.: Impact of the stratosphere on tropospheric climate change, Geophys. Res. Lett., 35, L12706, doi:10.1029/2008GL033573, 2008.

Song, I.-S. and Chun, H.-Y.: Momentum flux spectrum of convectively forced internal gravity waves and its application to gravity wave drag parameterization. Part I: Theory, J. Atmos. Sci., 62, 107-124, 2005.

Steck, T., Höpfner, M., von Clarmann, T., and Grabowski, U: Tomographic retrieval of atmospheric parameters from infrared limb emission observations, Appl. Optics, 44, 3291-3301, 2005. von Clarmann, T., De Clercq, C., Ridolfi, M., Höpfner, M., and Lambert, J.-C.: The horizontal resolution of MIPAS, Atmos. Meas. Tech., 2, 47-54, 2009, http://www.atmos-meas-tech.net/2/47/2009/.

Warner, C. D. and McIntyre, M. E.: An ultrasimple spectral parameterization for nonorographic gravity waves, J. Atmos. Sci., 58, 1837-1857, 2001.

Watanabe, S., Kawatani, Y., Tomikawa, Y., Miyazaki, K., Takahashi, M., and Sato, K.: General aspects of a T213L256 middle atmosphere general circulation model, J. Geophys. Res., 113, D12110, doi:10.1029/2008JD10026, 2008.

Wu, D. L. and Zhang, F.: A study of mesoscale gravity waves over the North Atlantic with satellite observations and a mesoscale model, J. Geophys. Res., 109, D22104, doi:10.1029/2004JD005090, 2004.

Wu, D. L. and Eckermann, S. D.: Global gravity wave variances from Aura MLS: Characteristics and interpretation, J. Atmos. Sci., 65, 3695-3718, 2008. 